\title{
LARGE-SCALE IRREGULARITIES IN THE INTERSTELLAR MEDIUM
}

\author{
Hari Om Vats \\ Physical Research Laboratory, India
}

\begin{abstract}
The radio flux measurement of the compact extragalactic objects are known to show two types of variablity i.e. intrinsic and extrinsic. Some of the unusual minima in the flux measurement seem to involve large structures in the interstellar medium. In this article we outline a simple ray approach for simulation of these minima in the flux measurement. Simulation will help in estimating the physical parameters of these large scale irregularities or structures in the interstellar medium. Some preliminary results of the simulation will also be described.
\end{abstract}

\section{THE MILKY WAY DISK WARP}

\author{
E. Florido ${ }^{1}$, E. Battaner ${ }^{1}$, E. Alfaro ${ }^{2}$ and M.L. Sanchez-Saavedra ${ }^{1}$ \\ ${ }^{1}$ Universidad de Granada, Spain \\ ${ }^{2}$ Instituto de Astrofísica de Andalucía, Spain
}

\begin{abstract}
A warped disk in our own galaxy is evident by means of HI, HII, $\gamma$-rays and dust observations, but unexistent when star distributions are considered, specially those of late type stars. This fact is in disagreement with the theories which assume a gravitational origin of warps, for instance a tidal interaction with the Magellanic Clouds. We tried to find the z-distribution of open clusters of different ages, for which a warp distribution was neither found nor rejected. Assuming an intergalactic magnetic field origin of the warp, we obtain a direction of the field in the Milky Way neighborhood given by $(b, 1)=\left(45^{\circ}, 74^{\circ}\right)$.
\end{abstract}

